\title{
A Reparameterization of PM6 Applied to Organic Diradical Molecules
}

\author{
Toru Saito*, ${ }^{*}$, Yasutaka Kitagawa ${ }^{\ddagger}, \S$, Yu Takano ${ }^{\dagger}$ \\ ${ }^{\dagger}$ Department of Biomedical Information Sciences, Graduate School of Information Sciences, \\ Hiroshima City University, 3-4-1 Ozuka-Higashi, Asa-Minami-Ku, Hiroshima 731-3194 \\ Japan \\ $\ddagger$ Division of Chemical Engineering, Department of Materials Engineering Science, Graduate \\ School of Engineering Science, Osaka University, 1-3 Machikaneyama, Toyonaka, Osaka \\ 560-0043 Japan \\ ${ }^{\S}$ Center for Spintronics Research Network (CSRN), Graduate School of Engineering \\ Science, Osaka University, Toyonaka, Osaka 560-8531, Japan \\ *e-mail: tsaito@hiroshima-cu.ac.jp
}

\section{Contents}

Section 1: Information about the training set (Table S1 and S2)

Section 2: Cartesian coordinates for 1-8 optimized by UrPM6

Section 3: Table S3

Section 4: Table S4

Section 5: Full citation for Ref. 65 


\section{Section1: Information about the training set}

Table S1: 25 species used in the subset I.

\begin{tabular}{|c|c|c|c|}
\hline \multicolumn{2}{|c|}{ closed-shell } & \multicolumn{2}{|c|}{ open-shell } \\
\hline $\mathrm{CO}$ & $(0,1)$ & $\mathrm{NH}$ & $(0,1$ and 3$)$ \\
\hline $\mathrm{H}_{2} \mathrm{O}$ & $(0,1)$ & $\mathrm{NH}_{2}^{+}$ & $(1,1$ and 3$)$ \\
\hline $\mathrm{N}_{2}$ & $(0,1)$ & $\mathrm{CH}_{2}$ & $(0,1$ and 3$)$ \\
\hline $\mathrm{NH}_{3}$ & $(0,1)$ & $\mathrm{O}_{3}$ & $(0,1)$ \\
\hline $\mathrm{CH}_{4}$ & $(0,1)$ & $\mathrm{CO}_{2}^{-}$ & $(0,2)$ \\
\hline $\mathrm{C}_{2} \mathrm{H}_{4}$ & $(0,1)$ & $\mathrm{CH}_{3}$ & $(0,2)$ \\
\hline $\mathrm{C}_{2} \mathrm{H}_{6}$ & $(0,1)$ & twisted $\mathrm{C}_{2} \mathrm{H}_{4}$ & $(0,1)$ \\
\hline $\mathrm{C}_{6} \mathrm{H}_{6}$ & $(0,1)$ & $\mathrm{H}_{2} \mathrm{NO}$ & $(0,2)$ \\
\hline $\mathrm{CH}_{3} \mathrm{OH}$ & $(0,1)$ & phenol radical & $(0,2)$ \\
\hline $\mathrm{CH}_{3} \mathrm{COO}^{-}$ & $(-1,1)$ & phenyl nitrene & $(0,1)$ \\
\hline imidazole & $(0,1)$ & pyridine radical & $(0,2)$ \\
\hline coronene & $(0,1)$ & $(a)^{b}$ & $(-1,2)$ \\
\hline & & $(b)^{b}$ & $(0,2)$ \\
\hline
\end{tabular}

${ }^{a}$ Values $(X, Y)$ represent charge and spin multiplicity, respectively.

${ }^{b}$ See Figure S1.

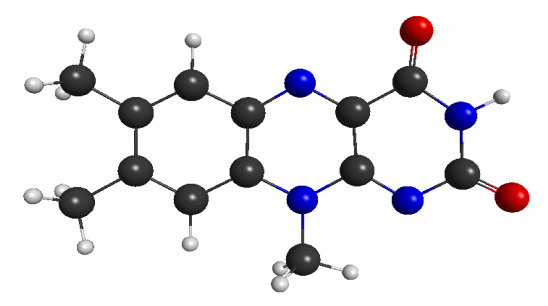

(a)

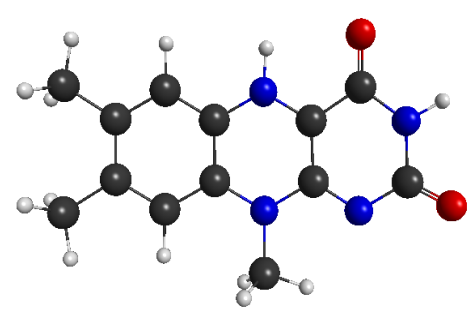

(b)

Figure. S 1: Truncated models for (a) flavin anion radical and (b) flavin radical 
Table S2: 8 reference data in the subset II consisting of bond dissociation energies, reaction energies, and singlet-triplet energy gaps.

\begin{tabular}{|c|c|c|c|}
\hline & & & Reference value (in kcal/mol) \\
\hline${ }^{1}$ oxyallyl & $\rightarrow$ & ${ }^{1}$ cyclopropanone & -30.0 \\
\hline $2{ }^{4} \mathrm{~N}$ & $\rightarrow$ & $\mathrm{N}_{2}$ & -222.86 \\
\hline${ }^{3} \mathrm{CH}_{2}$ & $\rightarrow$ & ${ }^{1} \mathrm{CH}_{2}$ & 6.0 \\
\hline${ }^{2} \mathrm{CH}_{3}+{ }^{2} \mathrm{H}$ & $\rightarrow$ & $\mathrm{CH}_{4}$ & -112.08 \\
\hline${ }^{3}$ trimethylenemethane & $\rightarrow$ & ${ }^{1}$ methylenecyclopropane & -22.04 \\
\hline${ }^{3}$ ortho-benzyne & $\rightarrow$ & ${ }^{1}$ ortho-benzyne & -29.5 \\
\hline${ }^{3}$ meta-benzyne & $\rightarrow$ & ${ }^{1}$ meta-benzyne & -11.7 \\
\hline${ }^{3}$ para-benzyne & & ${ }^{1}$ para-benzyne & -2.5 \\
\hline
\end{tabular}

Reference values were calculated at the UB3LYP/6-31G* level.

\section{Section2: Cartesian coordinates for 1-8 optimized by UrPM6}

1 (singlet)

C -0.7475700 .2001640 .727814$

$\mathrm{H}-1.6415070 .5398180 .225475$

H -0.8562140 .2238771 .802274$

1 (triplet)

C -0.9455570 .2719210 .840795$

$\mathrm{H}-1.5927490 .5241500 .068116$

H -0.7069850 .1677891 .846652$

2 (ortho-benzyne, singlet)

C -0.665890 1.1129700 .005089

C - $1.296673-0.0282040 .000355$

C -0.723934-1.282913 -0.002439

H - $1.271020-2.216787-0.006389$

C $0.694575-1.1915750 .000512$

H $1.269904-2.116435-0.001291$

C 1.3784590 .0456670 .005705

H 2.4676530 .0504620 .007796

C 0.7012361 .2954200 .008314

H 1.2010022 .2554490 .012258

2 (ortho-benzyne, triplet)

$\mathrm{C}-0.6904351 .1660600 .005350$

C - $1.354685-0.0356610 .000276$

C -0.701688 -1.244813 -0.002365
H $-1.242059-2.187769-0.006338$

C $0.717300-1.1966810 .000592$

H $1.276040-2.131090-0.001220$

C 1.3948710 .0291400 .005649

H 2.4833270 .0530630 .007824

C 0.6808081 .2563160 .008062

H 1.1918362 .2154900 .012080

2 (meta-benzyne, singlet)

C -4.294383 $3.906907-0.000015$

C -2.8808143 .9249490 .000400$

C $-2.2898305 .170551-0.000169$

C -2.831203 6.440864-0.001168

C $-4.2019816 .274707-0.001284$

C $-4.9853665 .140156-0.000789$

$\mathrm{H}-4.8390062 .9636540 .000422$

H -2.293549 3.0128100 .001161

H -2.291720 $7.375029-0.001479$

H -6.068927 5.192886 -0.000993

2 (meta-benzyne, triplet)

C -4.275644 3.9393760 .000004

C -2.861534 3.9216160 .000396

C $-2.2534525 .158967-0.000143$

C -2.850877 $6.406800-0.001160$

C $-4.2301966 .300434-0.001327$ 
C -4.997892 5.155174-0.000816

$\mathrm{H}-4.8205822 .9955360 .000441$

H -2.295971 2.9968410 .001136

H -2.309080 7.344954-0.001441

H -6.081551 5.182816-0.001005

2 (para-benzyne, singlet)

C -0.670461 1.1137870 .005129

C - $1.431452-0.0301110 .000062$

$\mathrm{H}-2.516444-0.026570-0.002005$

C -0.694694-1.252965-0.002286

$\mathrm{H}-1.198647-2.213818-0.006221$

C $0.672251-1.1148160 .000741$

C 1.4332430 .0290820 .005702

H 2.5182340 .0255410 .007757

C 0.6964841 .2519360 .008186

H 1.2004372 .2127890 .012162

2 (para-benzyne, triplet)

C -0.663289 1.1018830.005091

C - $1.437711-0.0402730 .000045$

$\mathrm{H}-2.522398-0.013127-0.001951$

C -0.706604 - $1.253748-0.002320$

H $-1.189545-2.225365-0.006265$

C $0.665079-1.1029120 .000750$

C 1.4395010 .0392440 .005775

H 2.5241880 .0120980 .007748

C 0.7083941 .2527190 .008200

H 1.1913352 .2243350 .012155

3 ( $D_{4 h}$, singlet)

with one imaginary frequency $\left(-318.8 i \mathrm{~cm}^{-1}\right)$

C 0.0000001 .0228220 .000000

C 1.0228220 .0000000 .000000

C $0.000000-1.0228220 .000000$

C - 1.0228220 .0000000 .000000

H 0.0000002 .0857640 .000000

H 2.0857640 .0000000 .000000

H $0.000000-2.0857640 .000000$

H -2.085764 0.0000000 .000000

3 ( $D_{4 h}$, triplet)

C 0.0000001 .0186700 .000000

C 1.0186700 .0000000 .000000

C $0.000000-1.0186700 .000000$

C - 1.0186700 .0000000 .000000

H 0.0000002 .0823360 .000000
H 2.0823360 .0000000 .000000

H $0.000000-2.0823360 .000000$

H -2.082336 0.0000000 .000000

3 ( $D_{2 h}$, closed-shell singlet)

C 0.0750151 .0250530 .000000

C 1.0250530 .0750150 .000000

C - $0.075015-1.0250530 .000000$

C - $1.025053-0.0750150 .000000$

H 0.0010262 .0866520 .000000

H 2.0866520 .0010260 .000000

H - $0.001026-2.0866520 .000000$

H $-2.086652-0.0010260 .000000$

4 (singlet)

C 0.000377 -1.7291810.000000

C -1.217067 - 1.036009 0.000000

$\mathrm{C}-1.2314640 .3858030 .000000$

C - 0.0004951 .0883880 .000000

C 1.2309030 .3865680 .000000

C $1.217388-1.0352570 .000000$

H $0.000712-2.8103530 .000000$

H -2.150066 - 1.5795070 .000000

$\mathrm{H}-0.0008322 .1682810 .000000$

H 2.150725 -1.578173 0.000000

C -2.475385 1.0997410 .000000

$\mathrm{H}-2.5022472 .1629770 .000000$

$\mathrm{H}-3.4067090 .5862200 .000000$

C 2.4743841 .1012780 .000000

H 3.4060250 .5883340 .000000

H 2.5005852 .1645300 .000000

4 (triplet)

C $0.000378-1.7331140 .000000$

C - $1.219106-1.0443500 .000000$

$\mathrm{C}-1.2458260 .4000850 .000000$

C - 0.0005031 .1061660 .000000

C 1.2452580 .4008590 .000000

C $1.219435-1.0435930 .000000$

H $0.000714-2.8147570 .000000$

H -2.152197 -1.584461 0.000000

$\mathrm{H}-0.0008382 .1841470 .000000$

H $2.152861-1.5831240 .000000$

C -2.461861 1.0924110 .000000

$\mathrm{H}-2.5043802 .1581840 .000000$

H -3.3998080 .5846990 .000000$

C 2.4608621 .0939400 .000000 
H 3.3991250 .5868100 .000000 H 2.5027212 .1597390 .000000

5 (singlet)

C $0.000009-0.045243-0.002198$

O -0.000107 - $1.299256-0.110585$

C -1.249059 0.7035580 .061628

$\mathrm{H}-2.1815360 .1593770 .013842$

H - 1.2703361 .7744130 .154260

C 1.2492150 .7031760 .063371

H 2.1815910 .1587100 .016885

H 1.2706901 .7740250 .156035

5 (triplet)

C 0.0000140 .0174080 .003187

O -0.000105 - $1.280265-0.109035$

C -1.2583220 .7006540 .061383$

H -2.171016 0.1255690 .011092

H -1.321111 1.7703100 .153753

C 1.2584770 .7002700 .063139

H 2.1710650 .1249060 .014122

H 1.3214641 .7699060 .155595

6 (singlet)

C $2.381040-2.9037940 .692815$

C $0.989763-2.5837420 .869203$

C $0.455067-1.3324570 .318953$

C $1.393418-0.2646300 .102228$

C $2.825303-0.5639190 .020080$

C $3.285535-1.9125290 .168691$

C - $0.944223-1.0940710 .066576$

C $0.9450941 .094732-0.063497$

C $-0.4541941 .333119-0.315913$

C -1.392549 0.265287 -0.099228

C $-2.8244410 .564569-0.017180$

C $-3.2847151 .913133-0.165998$

C $-2.3801442 .904457-0.689873$

C $-0.9888452 .584435-0.866120$

C $0.194117-3.4672851 .664053$

C -0.193112 3.468088 - 1.660771

C - $2.8629224 .182340-1.135556$

C $2.863885-4.1815901 .138658$

C $2.042583-5.0450831 .840608$

C - $0.7047824 .665913-2.137601$

C -2.041536 5.045942-1.837265

C $0.705852-4.6650382 .141005$

C -1.939498 -2.156355 -0.105604
C -3.333349 -1.847528 0.052517

C $-1.608148-3.483911-0.521973$

C $-4.313578-2.894974-0.018287$

C $-2.578355-4.463598-0.642975$

C $-3.957128-4.195832-0.349259$

C 1.9403622 .1569980 .108844

C 1.6089913 .4844900 .525399

C $3.3342191 .848178-0.049222$

C 2.5792094 .4641310 .646681

C 4.3144682 .8955800 .021906

C 3.9580124 .1963830 .353095

H 2.2924065 .4609560 .975547

H $5.3505982 .623295-0.167377$

H -2.415574 $6.007362-2.181510$

H $-3.9030214 .437926-0.926166$

H $-0.0853505 .320579-2.746320$

H $3.904001-4.4371630 .929315$

H 2.416708 -6.006403 2.185046

H $0.086500-5.3196032 .749915$

H -2.291563 -5.460480 -0.971668

H $-5.349668-2.6226920 .171179$

C $-4.980596-5.351634-0.447903$

C 4.9815465 .3520860 .452240

C $-4.517037-6.5046340 .475623$

H -5.245489 -7.318536 0.465429

$\mathrm{H}-3.553416-6.9070300 .155717$

$\mathrm{H}-4.410681-6.1476511 .503713$

C -5.036578 -5.828036 - 1.919656

H $-4.067942-6.211174-2.248366$

H -5.776236 -6.623145 -2.036590

H $-5.311756-4.998810-2.577376$

C $-6.397656-4.911187-0.010566$

$\mathrm{H}-6.391982-4.5466681 .019966$

H -6.782752 -4.122967 -0.662206

H $-7.086780-5.757993-0.065267$

C 6.3984564 .9118820 .014191

H 6.7837594 .1232440 .665220

H $6.3924624 .547991-1.016555$

H 7.0876295 .7586260 .069225

C $4.5178076 .505784-0.470330$

H $3.5544076 .908163-0.149743$

H $5.2464217 .319540-0.459889$

H $4.4109606 .149509-1.498610$

C 5.0379185 .8274171 .924321

H 4.0693206 .2101422 .253625

H 5.3134464 .9977502 .581344

H 5.7774916 .6225532 .041608 
C $3.7474730 .489333-0.181614$

C -3.746610 -0.488667 0.184665

H $4.7967760 .254965-0.359194$

H $-4.795888-0.2542580 .362319$

C $4.706136-2.269421-0.132385$

C $5.059964-2.700390-1.440522$

C $5.706499-2.1849420 .873121$

C $6.395451-3.040437-1.726766$

C $7.037970-2.5293620 .566427$

C $7.386438-2.956888-0.728453$

H $6.663138-3.369646-2.726269$

H $7.798000-2.4633821 .338955$

C -4.7054652 .2699460 .134492$

C -5.0606452 .7005731 .440762$

C -5.705608 2.185016 -0.873294

C -6.3980513 .0410311 .726516$

C -7.035563 2.529009 -0.567860

C -7.3862812 .9572930 .728435$

H -6.662989 3.3698892 .726827

H -7.797547 2.464222 -1.338615

C $5.356573-1.7285322 .262590$

H $4.946560-0.7092472 .228953$

H $4.579467-2.3798362 .686604$

H $6.224113-1.7370572 .927317$

C $8.807501-3.326477-1.055852$

H $8.860852-4.376149-1.369071$

H $9.180655-2.704043-1.877862$

H $9.469838-3.191376-0.195005$

C $-5.3529791 .728355-2.261977$

$\mathrm{H}-4.5761732 .380561-2.685140$

H $-4.9415270 .709692-2.227168$

H -6.219611 $1.735312-2.927902$

C -4.0196212 .7953442 .521608$

H -3.554877 1.8119812 .678992

H -3.2257973 .4901782 .215835$

H -4.442991 3.1393533 .468889

C -8.813806 3.3224521 .031845

H -8.945854 3.6011082 .082129

H -9.1255874 .1701070 .409723$

H -9.475989 2.475880 0.815115

C $4.016923-2.794509-2.519456$

H $3.223374-3.489050-2.212326$

H $3.552305-1.810919-2.675753$

H 4.438513 -3.138517 -3.467529

H 0.5686393 .7156760 .765218

H $0.8326593 .176859-1.899600$

H -0.831622 -3.176015 1.902972
H -0.567814 -3.715123 -0.761837

6 (triplet)

C $2.375691-2.9013670 .726361$

C $0.989521-2.5836580 .900445$

C $0.448360-1.3203320 .337503$

C $1.380684-0.2517140 .149286$

C $2.826565-0.5522180 .063650$

C $3.282931-1.9002200 .195088$

C -0.931989-1.094594 0.058499

C $0.9319901 .094597-0.058483$

C $-0.4483601 .320335-0.337487$

C $-1.3806830 .251717-0.149270$

C $-2.8265650 .552221-0.063634$

C $-3.2829301 .900223-0.195074$

C $-2.3756912 .901370-0.726346$

C $-0.9895212 .583661-0.900429$

C $0.196443-3.4475511 .702006$

C $-0.1964433 .447554-1.701990$

C -2.865325 4.160785 -1.191979

C $2.865325-4.1607831 .191994$

C $2.042994-5.0211501 .908217$

C - $0.7115584 .643771-2.198359$

C -2.042993 5.021153 -1.908202

C $0.711559-4.6437682 .198375$

C - $1.936132-2.173434-0.105219$

C $-3.324029-1.8655260 .045963$

C - $1.597839-3.491940-0.513417$

C $-4.302778-2.903139-0.033781$

C $-2.569609-4.478449-0.637501$

C -3.943089-4.210891 -0.358662

C 1.9361322 .1734370 .105235

C 1.5978403 .4919430 .513433

C $3.3240291 .865529-0.045947$

C 2.5696094 .4784520 .637518

C 4.3027782 .9031420 .033798

C 3.9430894 .2108940 .358679

H 2.2737565 .4758410 .956203

H $5.3417522 .632282-0.141221$

H -2.425491 $5.974266-2.266348$

H -3.907120 4.417208 -0.992515

H - $0.0868705 .289787-2.811036$

H $3.907120-4.4172060 .992528$

H $2.425491-5.9742632 .266363$

H $0.086871-5.2897832 .811052$

H - $2.273755-5.475839-0.956185$

H $-5.341752-2.6322790 .141238$ 
C -4.971951 -5.361810 -0.464853

C 4.9719525 .3618130 .464872

C -4.510504 -6.527360 0.443931

$\mathrm{H}-5.245164-7.3356420 .431108$

H -3.552504 -6.934108 0.112857

H -4.394004 -6.181395 1.474665

C -5.034643 -5.821593 - 1.941579

$\mathrm{H}-4.066715-6.199216-2.278854$

H -5.773720 -6.616364 -2.064098

H $-5.313885-4.985390-2.588644$

C -6.385387-4.920689-0.016768

$\mathrm{H}-6.372673-4.5628441 .016061$

H $-6.772525-4.127379-0.660957$

H -7.076951 -5.765403 -0.073125

C 6.3853864 .9206930 .016779

H 6.7725274 .1273810 .660964

H $6.3726684 .562852-1.016051$

H 7.0769505 .7654070 .073136

C $4.5105026 .527367-0.443906$

H $3.5525036 .934115-0.112825$

H $5.2451637 .335648-0.431082$

H $4.3939966 .181406-1.474640$

C 5.0346485 .8215891 .941600

H 4.0667216 .1992092 .278880

H 5.3138954 .9853832 .588660

H 5.7737256 .6163602 .064119

C $3.7391570 .493026-0.162924$

C $-3.739156-0.4930230 .162940$

H $4.7882350 .264410-0.347970$

H -4.788235 -0.264407 0.347985

C $4.697513-2.264344-0.119871$

C $5.033614-2.707087-1.428851$

C $5.710524-2.1766140 .872736$

C $6.364129-3.054621-1.728818$

C $7.036692-2.5289200 .552421$

C $7.367600-2.967517-0.743376$

H $6.618214-3.392428-2.729012$

H $7.806368-2.4604111 .315118$

C -4.697514 2.264346 0.119884

C -5.0336152 .7070901 .428865$

C -5.710524 2.176614 -0.872722

C -6.3641303 .0546171 .728832$

C -7.036694 2.528915 -0.552406

C -7.367603 2.967507 0.743391

H -6.618218 3.3924292 .729024

H -7.806367 2.460409 -1.315106

C $5.379547-1.7087292 .263074$
H $4.984899-0.6834732 .228145$

H $4.596223-2.3458992 .696987$

H $6.252617-1.7274662 .920322$

C $8.783096-3.345201-1.085305$

H $8.828294-4.396831-1.393183$

H $9.149180-2.729007-1.915173$

H $9.456104-3.208116-0.233094$

C -5.379546 $1.708732-2.263060$

$\mathrm{H}-4.5962172 .345898-2.696970$

H $-4.9849050 .683474-2.228134$

H -6.252614 $1.727476-2.920311$

C -3.9770522 .8041882 .494271$

H -3.5306601 .8150122 .667215$

H -3.173034 3.4755882 .163592

H - 4.3812323 .1772233 .438941

C -8.7830873 .3452281 .085326$

H -9.148871 2.7297381 .915846

H -8.8283684 .3971351 .392256$

H -9.456283 3.2072630 .233407

C $3.977052-2.804182-2.494257$

H $3.173037-3.475588-2.163584$

H $3.530656-1.815007-2.667195$

H $4.381233-3.177210-3.438930$

H 0.5557953 .7279530 .740067

H $0.8330063 .161971-1.931239$

H $-0.833005-3.1619671 .931256$

H $-0.555795-3.727951-0.740050$

7 (singlet)

H $6.2186951 .232128-3.524892$

C $5.5222460 .951975-2.724297$

C $6.0457430 .529789-1.493405$

C $3.2276380 .655532-1.888849$

C $5.1697030 .153288-0.419596$

C $4.1329831 .014326-2.923615$

C $3.7484350 .215015-0.606607$

C $5.699496-0.2832580 .836409$

H $3.7440661 .344075-3.886884$

C $4.834674-0.6426991 .876445$

H $5.261880-0.9739702 .831201$

C $3.438710-0.5800951 .697392$

H $2.792966-0.8663922 .527881$

C $2.872211-0.1594560 .475799$

C $1.414731-0.0870350 .259490$

H $0.847411-0.7658542 .231879$

C $0.495362-0.4306741 .255058$

C $-0.4953860 .430643-1.255105$ 
C -0.919213 -0.358233 1.037710

C $0.9191900 .358185-1.037764$

C - $1.4147540 .087024-0.259531$

C - $1.813125-0.7167772 .075535$

$\mathrm{H}-0.8474350 .765822-2.231927$

C $-3.227661-0.6556311 .888780$

$\mathrm{H}-1.420757-1.0464263 .036674$

C -3.748459 -0.2150230 .606569$

C -2.872236 $0.159498-0.475820$

C $1.8131030 .716673-2.075607$

H $1.4207361 .046275-3.036762$

C $7.5303730 .480651-1.301981$

C $7.181573-0.3649461 .042643$

N $8.0188870 .028762-0.041113$

C $9.475579-0.0349060 .152674$

C $12.242658-0.1559480 .521059$

C $10.142928-1.275094-0.047645$

C 10.1713381 .1456900 .534332

C 11.5680261 .0565420 .705199

C $11.538073-1.3080960 .153245$

H 12.1230241 .9495460 .985369

H $12.068255-2.2485340 .017398$

H $13.320573-0.2030950 .664547$

C 9.4542742 .4882720 .720530

H 8.3637532 .2885010 .841636

C $9.6542383 .338811-0.546205$

H $9.3290822 .767817-1.429928$

H $10.7060963 .599329-0.686915$

H $9.0737254 .260975-0.484443$

C 9.9301453 .2172511 .988412

H 10.9564363 .5781521 .887583

H 9.8858332 .5450342 .851595

H 9.2875644 .0796542 .184368

C $9.386943-2.553149-0.430316$

H $8.387290-2.261823-0.829873$

C $9.175143-3.4034200 .834692$

H $8.680511-2.7967211 .609249$

H $10.127333-3.7516001 .242342$

H $8.554154-4.2730250 .612566$

C $10.113200-3.334518-1.538184$

H $11.042574-3.781113-1.176948$

H $10.353871-2.670681-2.375065$

H $9.472554-4.140618-1.905370$

O $8.3210860 .826427-2.205755$

O $7.674282-0.7672202 .118048$

C -4.133004-1.014496 2.923523

H -3.744085 -1.344322 3.886764
C -5.522268 -0.952115 2.724215

$\mathrm{H}-6.218715-1.2323263 .524791$

C $-6.045765-0.5298191 .493362$

C -5.169727 -0.153246 0.419577

C -3.438737 $0.580247-1.697374$

$\mathrm{H}-2.7929950 .866587-2.527850$

C $-4.8347020 .642916-1.876403$

H $-5.2619080 .974282-2.831125$

C $-5.6995220 .283422-0.836384$

C -7.530395 -0.480639 1.301953

C -7.181599 0.365196-1.042584

N $-8.018913-0.0285820 .041148$

O $-7.6743100 .767604-2.117938$

O $-8.321106-0.8265232 .205690$

C -9.475605 $0.035188-0.152600$

C $-12.2426840 .156435-0.520918$

C -10.171443 - $1.145327-0.534370$

C -10.142877 1.2753940 .047866

C - $11.5380231 .308500-0.152993$

C - $11.568129-1.056076-0.705200$

H -12.068147 2.248954 -0.017031

H -12.123187 - $1.949018-0.985452$

H -13.320599 $0.203661-0.664380$

C $-9.454453-2.487930-0.720695$

H $-8.363953-2.288181-0.842030$

C -9.930592 -3.216941 - 1.988459

H $-9.287978-4.079280-2.184592$

H $-9.886575-2.544716-2.851649$

H - $10.956820-3.577949-1.887355$

C $-9.654187-3.3384280 .546105$

H $-9.073715-4.2606110 .484255$

H -10.706025 -3.598907 0.687031

H -9.328843 -2.767413 1.429750

C -9.3868092 .5533620 .430662$

H -8.387160 2.261936 0.830157

C -10.112990 3.3346441 .538641

H -10.353657 2.670727 2.375459

H -11.042360 3.7813151 .177489

H -9.472297 4.1406791 .905889

C $-9.1749983 .403767-0.834255$

H -8.680420 2.797128 -1.608895

H -8.553954 $4.273312-0.612047$

H -10.127180 $3.752045-1.241839$

7 (triplet)

H $6.2243891 .233512-3.529496$

C $5.5198020 .955552-2.734939$ 
C $6.0417450 .531115-1.497401$ C $3.2141890 .659003-1.898568$ C $5.1624960 .154015-0.422421$ C $4.1402481 .019767-2.939171$ C $3.7398240 .215968-0.609670$ C $5.690499-0.2811430 .828931$ H $3.7479791 .349024-3.901085$ C $4.822544-0.6419891 .872281$ H $5.252915-0.9728842 .825541$ C $3.432409-0.5789291 .692028$ H $2.781440-0.8641852 .518896$ C $2.867261-0.1562020 .465487$ C $1.407105-0.0831780 .247900$ H $0.844637-0.7644752 .226064$ C $0.483575-0.4300151 .252230$ C $-0.4835950 .430181-1.252166$ C -0.909644 -0.355428 1.029507 C $0.9096240 .355596-1.029443$ C - $1.4071250 .083344-0.247837$ C -1.829240 -0.722044 2.090535 H $-0.8446580 .764641-2.226000$ C -3.214210 - 0.6588251 .898636 H -1.418459 -1.049202 3.044027 C -3.739844 -0.215794 0.609736 C $-2.8672810 .156368-0.465424$ C $1.8292190 .722217-2.090469$ H $1.4184391 .049376-3.043961$ C $7.5252560 .481611-1.304139$ C $7.171949-0.3639811 .038984$ N $8.0119910 .029454-0.043090$ C $9.468291-0.0346440 .152826$ C $12.234718-0.1565970 .525452$ C $10.135491-1.275097-0.046384$ C 10.1638291 .1457500 .535530 C 11.5602211 .0561310 .708514 C $11.530314-1.3085340 .156645$ H 12.1150911 .9489470 .989531 H $12.060386-2.2491650 .021710$ H $13.312396-0.2040990 .670592$ C 9.4468842 .4885360 .720727 H 8.3561962 .2890430 .840664 C $9.6485143 .338871-0.545871$ H $9.3242182 .767986-1.429928$ H $10.7005993 .599088-0.685392$ H $9.0682404 .261229-0.484825$ C 9.9214893 .2175681 .989032 H 10.9479153 .5783521 .889297
H 9.8761412 .5454712 .852243 H 9.2787954 .0800552 .184221 C $9.379636-2.552933-0.430053$ H $8.380888-2.261286-0.831582$ C $9.165072-3.4026500 .834839$ H $8.669064-2.7955701 .608207$ H $10.116315-3.7510001 .244514$ H $8.544236-4.2721320 .611818$ C $10.107607-3.335033-1.536262$ H $11.035954-3.782140-1.173046$ H $10.350375-2.671645-2.372861$ H $9.467195-4.140847-1.904467$ O $8.3164600 .827797-2.207933$ O $7.662582-0.7666422 .115084$ C -4.140268-1.019580 2.939242 H $-3.748000-1.3488343 .901157$ C -5.519822 -0.955359 2.735012 H -6.224410 - 1.2333113 .529572 C -6.041765 -0.530927 1.497472 C -5.162517 -0.153838 0.422488 C -3.432429 0.579088 - 1.691967 $\mathrm{H}-2.7814600 .864337-2.518837$ C $-4.8225640 .642149-1.872219$ H -5.252935 $0.973038-2.825481$ C -5.690520 $0.281313-0.828866$ C -7.525277-0.481415 1.304213 C -7.171969 0.364154-1.038919 $\mathrm{N}-8.012011-0.0292680 .043160$ O $-7.6626020 .766805-2.115022$ O $-8.316480-0.8275872 .208012$ C -9.468311 $0.034838-0.152753$ C -12.234739 $0.156803-0.525373$ C -10.163858 - $1.145556-0.535442$ C -10.135503 1.2752970 .046445 C -11.530326 1.308740 -0.156581 C - $11.560250-1.055931-0.708424$ H -12.060393 2.249376 -0.021655 H - $12.115125-1.948747-0.989430$ H -13.312417 $0.204310-0.670512$ C $-9.446922-2.488349-0.720627$ H $-8.356232-2.288864-0.840569$ C $-9.921534-3.217393-1.988922$ H $-9.278845-4.079884-2.184104$ H $-9.876187-2.545305-2.852140$ H -10.947961 -3.578171 - 1.889179 C -9.648552 -3.338669 0.545982 H $-9.068284-4.2610310 .484945$ 
H -10.700639 -3.598878 0.685508

$\mathrm{H}-9.324251-2.7677751 .430031$

C -9.379640 2.5531320 .430099

H -8.380894 2.261484 0.831634

C -10.107607 3.3352531 .536296

H -10.350383 2.671878 2.372902

H -11.035947 3.7823651 .173071

H -9.467187 4.1410651 .904492

C $-9.1650673 .402830-0.834804$

$\mathrm{H}-8.6690612 .795736-1.608163$

H -8.544227 $4.272311-0.611795$

H -10.116307 $3.751180-1.244487$

7 (closed-shell singlet)

H $6.2104991 .231740-3.511635$

C $5.5266580 .947125-2.701511$

C $6.0544400 .527766-1.482745$

C $3.2469220 .649899-1.868118$

C $5.1820020 .149387-0.406890$

C $4.1237591 .006840-2.893859$

C $3.7656170 .209626-0.589595$

C $5.717376-0.2895540 .852485$

H $3.7396731 .338087-3.858603$

C $4.860583-0.6478181 .885796$

H $5.281604-0.9811372 .843112$

C $3.452469-0.5857041 .708733$

H $2.817989-0.8753762 .546407$

C $2.892140-0.1689970 .501974$

C $1.418656-0.0969190 .287635$

H $0.849321-0.7687392 .240033$

C $0.520722-0.4304201 .254232$

C - $0.5207350 .430151-1.254315$

C -0.935294 -0.361310 1.046407

C $0.9352800 .361309-1.046398$

C - $1.4186680 .096585-0.287740$

C - $1.793076-0.7070562 .046873$

$\mathrm{H}-0.8493340 .768344-2.240159$

C -3.246938 -0.649041 1.868423

H -1.427881 -1.040713 3.019479

C $-3.765631-0.2094640 .589659$

C - $2.8921520 .168423-0.502162$

C $1.7930610 .707671-2.046652$

H $1.4278651 .041649-3.019147$

C $7.5387640 .479817-1.295473$

C $7.200173-0.3687271 .050971$

N $8.0316950 .027129-0.035828$

C $9.489262-0.0350650 .152700$
C $12.257961-0.1532550 .510964$

C $10.157440-1.274236-0.051088$

C 10.1851911 .1459400 .532760

C 11.5825941 .0582950 .698533

C $11.553343-1.3058770 .144707$

H 12.1375691 .9516930 .977349

H $12.084082-2.2455570 .006118$

H $13.336399-0.1992870 .650504$

C 9.4672592 .4875800 .722271

H 8.3774642 .2863210 .847562

C $9.6611513 .338615-0.545081$

H $9.3335082 .766780-1.427501$

H $10.7120583 .600626-0.689809$

H $9.0794044 .259784-0.481182$

C 9.9471933 .2168491 .988486

H 10.9726883 .5788601 .883719

H 9.9070062 .5442802 .851587

H 9.3044284 .0784532 .187113

C $9.401447-2.552784-0.432034$

H $8.400229-2.262045-0.828164$

C $9.194546-3.4038530 .833272$

H $8.702453-2.7974021 .609768$

H $10.148364-3.7516161 .237396$

H $8.573194-4.2735930 .613007$

C $10.124748-3.332935-1.542716$

H $11.055847-3.778607-1.184852$

H $10.361812-2.668314-2.380019$

H $9.483729-4.139498-1.908117$

O $8.3281920 .826581-2.199838$

O $7.698145-0.7712632 .124163$

C -4.123776 -1.005176 2.894443

H $-3.739692-1.3358743 .859375$

C $-5.526677-0.9453452 .702137$

$\mathrm{H}-6.210520-1.2292923 .512493$

C -6.054457 -0.526741 1.483110

C -5.182016 -0.1492170.406956

C -3.452479 $0.584259-1.709223$

$\mathrm{H}-2.8179980 .873275-2.547123$

C $-4.8605920 .646299-1.886315$

$\mathrm{H}-5.2816110 .978919-2.843875$

C $-5.7173870 .288861-0.852720$

C -7.538784 -0.478705 1.295878

C -7.200183 0.367993-1.051232

N -8.031709 -0.026928 0.035904

O $-7.6981510 .769716-2.124730$

O $-8.328219-0.8246402 .200554$

C $-9.4892760 .035289-0.152618$ 
C -12.257978 $0.153511-0.510851$

C -10.185363 - $1.145946-0.531668$

C -10.157297 1.2747080 .050177

C -11.553204 $1.306353-0.145586$

C $-11.582764-1.058275-0.697444$

H -12.083825 2.246211 -0.007754

H -12.137857-1.951839-0.975490

H -13.336417 $0.199556-0.650378$

C $-9.467605-2.487829-0.720110$

$\mathrm{H}-8.377778-2.286819-0.845524$

C $-9.947604-3.218014-1.985773$

H $-9.304952-4.079860-2.183716$

H -9.907301 -2.546119-2.849393

H -10.973152 -3.579801 -1.880754

C $-9.661648-3.3378500 .547900$

$\mathrm{H}-9.080006-4.2591380 .484744$

H -10.712591 -3.599624 0.692793

H $-9.333967-2.7653621 .429883$

C -9.401142 2.5534790.430049

$\mathrm{H}-8.3999302 .2629500 .826349$

C -10.124289 3.3345951 .540153

H -10.361377 2.670666 2.377998

H -11.055360 3.7800851 .181990

H -9.483157 4.1413761 .904873

C -9.194224 $3.403517-0.835947$

H -8.702234 2.796399 -1.611983

H -8.572772 $4.273370-0.616409$

H -10.148028 3.751054-1.240296

8 ( $n=2$, singlet $)$

C $-0.000002-2.4786480 .715813$

C $0.000002-2.478648-0.715813$

C $0.000001-3.664407-1.416295$

C $-0.000001-3.6644071 .416295$

C $0.000000-4.9218140 .716115$

C $0.000000-4.921814-0.716115$

H $0.000002-3.674735-2.504200$

H $-0.000002-3.6747352 .504200$

C $-0.000001-6.179928-1.416131$

C $0.000002-6.1799281 .416131$

C -0.000001 -7.365324-0.715986

H $-0.000002-8.319948-1.238588$

C $0.000001-7.3653240 .715986$

H $0.000001-8.3199481 .238588$

H -0.000002 -6.169944 -2.504150

H $0.000002-6.1699442 .504150$

H $0.000002-1.523993-1.238589$
H -0.000003 - 1.5239931 .238589

8 ( $n=2$, triplet $)$

C $0.000001-2.4374010 .680345$

C $-0.000001-2.437401-0.680345$

C $0.000000-3.687628-1.421983$

C $0.000000-3.6876281 .421983$

C $0.000000-4.9194370 .742479$

C $0.000000-4.919437-0.742479$

H $-0.000001-3.651357-2.505750$

H $0.000001-3.6513572 .505750$

C $0.000001-6.156795-1.423585$

C $0.000000-6.1567951 .423585$

C $0.000000-7.408394-0.679419$

H $0.000001-8.331709-1.254887$

C $0.000000-7.4083940 .679419$

H - $0.000001-8.3317091 .254887$

H $0.000001-6.192576-2.506672$

H -0.000001 -6.192576 2.506672

H - $0.000001-1.513444-1.254463$

H $0.000001-1.5134441 .254463$

8 ( $n=3$, singlet)

C $0.000000-0.0170610 .722353$

C $0.000000-0.017061-0.722353$

C $0.000000-1.192155-1.420560$

C $0.000000-1.1921551 .420560$

C $0.000000-2.4678240 .723163$

C $0.000000-2.467824-0.723163$

H $0.000000-1.2063942 .508465$

H $0.000000-1.206394-2.508465$

C $0.000000-3.693114-1.420293$

C $0.000000-3.6931141 .420293$

C $0.000000-4.9183980 .723165$

C $0.000000-4.918398-0.723165$

H $0.000000-3.693111-2.508431$

H $0.000000-3.6931112 .508431$

C $0.000000-6.194072-1.420562$

C $0.000000-6.1940721 .420562$

C $0.000000-7.369162-0.722355$

H $0.000000-8.328161-1.236343$

C $0.000000-7.3691620 .722355$

H $0.000000-8.3281611 .236343$

H $0.000000-6.179829-2.508467$

H $0.000000-6.1798292 .508467$

H $0.0000000 .941935-1.236347$

H 0.0000000 .9419351 .236347 
8 ( $n=3$, triplet $)$

C 0.0000000 .0149030 .692908

C $0.0000000 .014903-0.692908$

C $0.000000-1.212017-1.412557$

C $0.000000-1.2120171 .412557$

C $0.000000-2.4313360 .727032$

C $0.000000-2.431336-0.727032$

H $0.000000-1.1908772 .499684$

H $0.000000-1.190877-2.499684$

C $0.000000-3.693112-1.439778$

C $0.000000-3.6931121 .439778$

C $0.000000-4.9548880 .727031$

C $0.000000-4.954888-0.727031$

H $0.000000-3.693113-2.522041$

H $0.000000-3.6931132 .522041$

C $0.000000-6.174208-1.412557$

C $0.000000-6.1742081 .412557$

C $0.000000-7.401127-0.692909$

H $0.000000-8.335979-1.249663$

C $0.000000-7.4011270 .692909$

H $0.000000-8.3359791 .249663$

H $0.000000-6.195347-2.499685$

H $0.000000-6.1953472 .499685$

H $0.0000000 .949755-1.249663$

H 0.0000000 .9497551 .249663

8 ( $n=4$, singlet $)$

C $0.0000002 .447959-0.723463$

C 0.0000002 .4479590 .723463

C 0.0000001 .2746241 .422560

H 0.0000001 .2610712 .510659

C $0.000000-0.0052790 .727568$

C $0.000000-0.005279-0.727568$

C $0.0000001 .274624-1.422560$

H $0.0000001 .261071-2.510659$

C $0.000000-1.220511-1.423652$

C $0.000000-1.2205111 .423652$

C $0.000000-2.4645000 .726143$

C $0.000000-2.464500-0.726143$

H $0.000000-1.2256372 .511597$

H $0.000000-1.225637-2.511597$

C $0.000000-3.708491-1.423652$

C $0.000000-3.7084911 .423652$

C $0.000000-4.9237220 .727568$

C $0.000000-4.923722-0.727568$

H $0.000000-3.703364-2.511597$
H $0.000000-3.7033642 .511597$

C $0.000000-6.203626-1.422560$

C $0.000000-6.2036261 .422560$

C $0.000000-7.376960-0.723464$

H $0.000000-8.337202-1.235540$

C $0.000000-7.3769600 .723464$

H $0.000000-8.3372021 .235540$

H $0.000000-6.190073-2.510659$

H $0.000000-6.1900732 .510659$

H $0.0000003 .408201-1.235540$

H 0.0000003 .4082011 .235540

$8(n=4$, triplet $)$

C $0.0000002 .470267-0.702720$

C 0.0000002 .4702670 .702720

C 0.0000001 .2620451 .412114

H 0.0000011 .2680612 .499962

C 0.0000000 .0308300 .717864

C $0.0000000 .030830-0.717864$

C $0.0000001 .262045-1.412114$

H -0.000001 $1.268061-2.499962$

C $0.000000-1.238114-1.433617$

C $0.000000-1.2381141 .433617$

C $0.000000-2.4645010 .746005$

C $0.000000-2.464501-0.746005$

H $0.000000-1.2157422 .519163$

H $0.000000-1.215742-2.519163$

C $0.000000-3.690887-1.433617$

C $0.000000-3.6908871 .433617$

C $0.000000-4.9598310 .717864$

C $0.000000-4.959831-0.717864$

H $0.000000-3.713259-2.519163$

H $0.000000-3.7132592 .519163$

C $0.000000-6.191046-1.412114$

C $0.000000-6.1910461 .412114$

C $0.000000-7.399268-0.702720$

H $0.000000-8.342985-1.244696$

C $0.000000-7.3992680 .702720$

H $0.000000-8.3429851 .244696$

H $0.000000-6.197062-2.499962$

H $0.000000-6.1970622 .499962$

H $0.0000003 .413984-1.244697$

H 0.0000013 .4139841 .244697

8 ( $n=5$, singlet)

C -0.000001 4.924105 0.720081

C $0.0000004 .924105-0.720081$ 
C -0.0000013.744303 1.421018

C $0.0000013 .744303-1.421018$

C $0.0000002 .472350-0.726322$

C 0.0000002 .4723500 .726322

C 0.0000001 .2485741 .425816

$\mathrm{H}-0.0000011 .2474462 .513474$

C 0.0000000 .0060420 .730632

C $0.0000000 .006042-0.730632$

C $0.0000001 .248574-1.425816$

H -0.000001 3.7341072 .509086

H $0.0000013 .734107-2.509086$

H $0.0000011 .247446-2.513474$

C $0.000000-1.230885-1.428078$

C $0.000000-1.2308851 .428078$

C $0.000000-2.4677620 .730635$

C $0.000000-2.467762-0.730635$

H $0.000000-1.2308582 .515362$

H $0.000000-1.230858-2.515362$

C $0.000000-3.710340-1.425817$

C $0.000001-3.7103401 .425817$

C $0.000000-4.9340810 .726333$

C $0.000000-4.934081-0.726333$

H -0.000001 -3.709166 -2.513476

H $0.000001-3.7091662 .513476$

C $-0.000001-6.206060-1.421023$

C $0.000001-6.2060601 .421023$

C $0.000000-7.385848-0.720091$

H $-0.000001-8.343104-1.237435$

C $0.000000-7.3858480 .720091$

H $0.000000-8.3431041 .237435$

H $-0.000001-6.195844-2.509093$

H $0.000001-6.1958442 .509093$

H $0.0000015 .881347-1.237450$

H -0.000001 5.8813471 .237450

$8(n=5$, triplet $)$

C 0.0000004 .9341420 .710331

C $0.0000174 .934142-0.710331$

C -0.000012 3.7395361 .413970

C $0.0000193 .739536-1.413970$

C $0.0000052 .494067-0.715314$

C -0.000008 2.494067 0.715314

C -0.000014 1.2313231 .425937

$\mathrm{H}-0.0000211 .2543672 .513360$

C -0.0000100 .0254870 .739847$

C $-0.0000050 .025487-0.739847$

C $0.0000041 .231323-1.425937$
H -0.000024 3.7352972 .501974

H $0.0000313 .735297-2.501974$

H $0.0000121 .254367-2.513360$

C -0.000009 -1.230869 -1.439364

C -0.000009-1.230869 1.439364

C $-0.000006-2.4872260 .739848$

C $-0.000011-2.487226-0.739848$

H $-0.000008-1.2308702 .524776$

H $-0.000008-1.230870-2.524776$

C -0.000015 -3.693064-1.425939

C $0.000003-3.6930641 .425939$

C $0.000004-4.9558100 .715314$

C $-0.000009-4.955810-0.715314$

H $-0.000022-3.716113-2.513363$

H $0.000011-3.7161132 .513363$

C -0.000013 -6.201279 -1.413972

C $0.000019-6.2012791 .413972$

C $0.000001-7.395885-0.710330$

H $-0.000001-8.346056-1.240956$

C $0.000018-7.3958850 .710330$

H $0.000029-8.3460561 .240956$

H $-0.000024-6.197038-2.501975$

H $0.000031-6.1970382 .501975$

H $0.0000275 .884315-1.240953$

H -0.000003 5.8843151 .240953

8 ( $n=6$, singlet $)$

C 0.0000007 .3941250 .718896

C 0.0000006 .2125381 .420282

C 0.0000004 .9437470 .725011

C $0.0000004 .943747-0.725011$

C $0.0000007 .394125-0.718896$

C 0.0000003 .7155271 .425693

C $0.0000003 .715527-1.425693$

C $0.0000002 .477757-0.730882$

C 0.0000002 .4777570 .730882

C 0.0000001 .2366231 .429374

H 0.0000001 .2389442 .516781

C 0.0000000 .0000000 .733768

C $0.0000000 .000000-0.733768$

C $0.0000001 .236623-1.429374$

H 0.0000003 .7171882 .513444

H 0.0000008 .3506221 .237999

H 0.0000006 .2032682 .508436

H $0.0000006 .203268-2.508436$

H $0.0000008 .350622-1.237999$

H $0.0000003 .717188-2.513444$ 
H $0.0000001 .238944-2.516781$

C $0.000000-1.236623-1.429374$

C $0.000000-1.2366231 .429374$

C $0.000000-2.4777570 .730882$

C $0.000000-2.477757-0.730882$

H $0.000000-1.2389442 .516781$

H $0.000000-1.238944-2.516781$

C $0.000000-3.715527-1.425693$

C $0.000000-3.7155271 .425693$

C $0.000000-4.9437470 .725011$

C $0.000000-4.943747-0.725011$

H $0.000000-3.717188-2.513444$

H $0.000000-3.7171882 .513444$

C $0.000000-6.212538-1.420282$

C $0.000000-6.2125381 .420282$

C $0.000000-7.394125-0.718896$

H $0.000000-8.350622-1.237999$

C $0.000000-7.3941250 .718896$

H $0.000000-8.3506221 .237999$

H $0.000000-6.203268-2.508436$

H $0.000000-6.2032682 .508436$

C $0.0000006 .212538-1.420282$

8 ( $n=6$, triplet $)$

C 0.0000007 .3972170 .715253

C 0.0000006 .2107651 .416139

C 0.0000004 .9537790 .716793

C $0.0000004 .953779-0.716793$

C $0.0000007 .397217-0.715253$

C 0.0000003 .7029871 .422076

C $0.0000003 .702987-1.422076$

C $0.0000002 .497119-0.731951$

C 0.0000002 .4971190 .731951

C 0.0000001 .2251911 .435807

H 0.0000001 .2415322 .522634

C 0.0000000 .0003730 .748152

C $0.0000000 .000373-0.748152$

C $0.0000001 .225191-1.435807$

H 0.0000003 .7184322 .510261

H 0.0000008 .3511031 .239256

H 0.0000006 .2017502 .504233

H $0.0000006 .201750-2.504233$

H $0.0000008 .351103-1.239256$

H $0.0000003 .718432-2.510261$

H $0.0000001 .241532-2.522634$

C $0.000000-1.225472-1.436035$

C $0.000000-1.2254721 .436035$
C $0.000000-2.4971300 .732245$

C $0.000000-2.497130-0.732245$

H $0.000000-1.2414532 .522820$

H $0.000000-1.241453-2.522820$

C $0.000000-3.702742-1.422142$

C $0.000000-3.7027421 .422142$

C $0.000000-4.9540860 .716680$

C $0.000000-4.954086-0.716680$

H $0.000000-3.718530-2.510318$

H $0.000000-3.7185302 .510318$

C $0.000000-6.210608-1.416035$

C $0.000000-6.2106081 .416035$

C $0.000000-7.397366-0.715065$

H $0.000000-8.351105-1.239334$

C $0.000000-7.3973660 .715065$

H $0.000000-8.3511051 .239334$

H $0.000000-6.201758-2.504128$

H $0.000000-6.2017582 .504128$

C $0.0000006 .210765-1.416139$

8 ( $n=8$, singlet $)$

C -0.001849 7.374971 0.712098

C -0.0004466 .1944871 .415035$

C -0.0001774 .9248580 .721280$

C -0.001244 4.922820 -0.728028

C -0.003031 7.372950 -0.725729

C 0.0010023 .6969381 .423660

C -0.000889 3.692929-1.426953

C $0.0003102 .458107-0.730091$

C 0.0011312 .4601620 .730272

C 0.0019071 .2165381 .430799

H 0.0024341 .2221022 .518338

C $0.001882-0.0158010 .737137$

C $0.001444-0.017866-0.729985$

C $0.0006511 .212516-1.427114$

H 0.0017603 .7003802 .511471

H - 0.0020928 .3322511 .229833

H 0.0004646 .1865632 .503218

H -0.003567 6.179514 -2.513503

H $-0.0041808 .328771-1.246150$

H $-0.0015933 .693313-2.514769$

H $0.0002231 .215018-2.514664$

C $0.001725-1.261641-1.424866$

C $0.002132-1.2576141 .435519$

C $0.001980-2.4951550 .741165$

C $0.002011-2.497222-0.727028$

H $0.002349-1.2547502 .523106$ 
H $0.001636-1.261842-2.512457$

C $0.002203-3.734761-1.421382$

C $0.001678-3.7307331 .439003$

C $0.001449-4.9745110 .744122$

C $0.001949-4.976576-0.723000$

H $0.002460-3.737626-2.508969$

H $0.001538-3.7305342 .526594$

C $0.002040-6.208913-1.416662$

C $0.000659-6.2048921 .441251$

C $0.001298-7.452538-0.716135$

C $0.000410-7.4504840 .744227$

H $0.002606-6.214478-2.504201$

H $0.000175-6.2073942 .528801$

C -0.002706 6.190495-1.425347

C $0.000180-9.917234-0.707143$

C $-0.000959-9.9151960 .742165$

C $0.001286-8.689313-1.409524$

H $0.002090-8.692755-2.497334$

C - $0.000742-8.6853051 .441090$

$\mathrm{H}-0.001505-8.6856882 .528906$

C -0.002329 -11.182870 1.439482

C $-0.002482-12.3653270 .739864$

H - $0.003549-13.3211461 .260288$

C $-0.001225-12.367348-0.697962$

H -0.001331 -13.324626 -1.215698

C $0.000077-11.186862-1.400898$

H $0.001038-11.178938-2.489081$

H -0.003253 -11.171891 2.527638

8 ( $n=8$, triplet)

C -0.000090 7.370847 0.711649

C -0.0000776 .1965271 .414164$

C - 0.0000314 .9212950 .720436

C $0.0000024 .916820-0.724274$

C $-0.0000577 .366109-0.730887$

C - 0.0000183 .6954411 .422481

C $0.0000483 .685522-1.419133$

C $0.0000622 .465349-0.720084$

C 0.0000282 .4684230 .729505

C 0.0000421 .2056851 .434072

H 0.0000161 .2243782 .522152

C $0.000088-0.0002430 .746827$

C $0.0001230 .000964-0.730031$

C $0.0001091 .193066-1.418994$

H -0.000043 3.7002312 .510842

H -0.000124 8.3311211 .223942

H -0.000102 6.1864792 .502375
H $0.0000126 .170353-2.514075$

$\mathrm{H}-0.0000698 .323014-1.249482$

H $0.0000733 .685088-2.507572$

H $0.0001341 .211481-2.507467$

C $0.000171-1.279758-1.428333$

C $0.000102-1.2618141 .445713$

C $0.000149-2.4987340 .756551$

C $0.000185-2.493867-0.741889$

H $0.000076-1.2523992 .532942$

H $0.000196-1.262505-2.515872$

C $0.000232-3.730309-1.430976$

C $0.000163-3.7126641 .442943$

C $0.000211-4.9933150 .744592$

C $0.000246-4.992124-0.732043$

H $0.000258-3.739920-2.518216$

H $0.000137-3.7300072 .530493$

C $0.000292-6.197869-1.419492$

C $0.000224-6.1857721 .433509$

C $0.000306-7.460624-0.715119$

C $0.000272-7.4577160 .734494$

H $0.000318-6.216393-2.507581$

H $0.000199-6.2041732 .521973$

C $-0.0000136 .187135-1.425941$

C $0.000366-9.913508-0.706548$

C $0.000332-9.9092890 .738226$

C $0.000352-8.687580-1.408353$

H $0.000378-8.692136-2.496713$

C $0.000286-8.6782131 .433328$

H $0.000261-8.6779562 .521760$

C $0.000347-11.1798061 .439578$

C $0.000392-12.3586160 .744255$

H $0.000403-13.3156421 .262632$

C $0.000425-12.363066-0.698297$

H $0.000460-13.323241-1.210779$

C $0.000413-11.188597-1.400551$

H $0.000437-11.178320-2.488761$

H $0.000322-11.1632982 .527717$

8 ( $n=10$, singlet $)$

C 0.0000177 .3810310 .729081

C 0.0001726 .1527141 .430090

C 0.0002234 .9163080 .735353

C $0.0001544 .915791-0.724959$

C $-0.0000757 .380517-0.720433$

C 0.0003173 .6722721 .434504

C $0.0002143 .671260-1.423229$

C $0.0002912 .440179-0.727222$ 
C 0.0003252 .4406980 .739368 C 0.0003401 .1975531 .436405 H 0.0003601 .1994602 .524041 C $0.000306-0.0374300 .740399$ C $0.000314-0.037949-0.726500$ C $0.0003131 .196542-1.423379$ H 0.0003653 .6765132 .522065 H 0.0002396 .1548372 .517914 H - $0.0000486 .153057-2.508397$ H $0.0001853 .674732-2.510793$ H $0.0003151 .197679-2.511016$ C $0.000301-1.278081-1.422363$ C $0.000247-1.2770701 .437139$ C $0.000192-2.5149000 .741056$ C $0.000239-2.515418-0.725404$ H $0.000233-1.2764252 .524857$ H $0.000331-1.278206-2.510080$ C $0.000214-3.753249-1.421488$ C $0.000087-3.7522371 .438015$ C $0.000047-4.9923690 .742151$ C $0.000127-4.992888-0.724747$ H $0.000261-3.753893-2.509205$ H $0.000038-3.7521122 .525732$ C $0.000125-6.227872-1.420753$ C -0.000062 -6.226860 1.439031 C $0.000058-7.471016-0.723716$ C $-0.000050-7.4704970 .742873$ H $0.000188-6.229778-2.508389$ $\mathrm{H}-0.000141-6.2279972 .526667$ C $0.0000096 .151704-1.420572$ C $0.000097-9.946626-0.719701$ C - $0.000029-9.9461090 .740611$ C $0.000116-8.702590-1.418852$ H $0.000196-8.706832-2.506413$ C $-0.000116-8.7015791 .438881$ $\mathrm{H}-0.000212-8.7050502 .526445$ C $-0.000026-11.1820231 .436224$ C $0.000142-12.4108350 .736085$ C $0.000274-12.411349-0.713429$ C $0.000232-11.183032-1.414438$ H $0.000321-11.185155-2.502262$ H -0.000136 -11.183374 2.524049 C $0.000561-14.861553-0.706799$ H $0.000731-15.818363-1.225430$ C $0.000431-14.8610440 .731193$ H $0.000508-15.8174851 .250501$ C $0.000472-13.680469-1.408484$
C $0.000214-13.6794621 .432040$ H $0.000562-13.671363-2.496667$ H $0.000108-13.6695842 .520216$ C -0.000266 8.649144-1.416388 C -0.000360 9.830725 -0.715541 H - $0.00051310 .787167-1.234849$ C -0.000068 8.650151 1.424136 H 0.0000148 .6410442 .512318 C -0.0002569.831235 0.722451 H - 0.00032910 .7880441 .241081 H -0.000338 $8.639266-2.504564$

$8(n=10$, triplet $)$

C 0.0001727 .3777860 .732887

C 0.0000916 .1490321 .429710

C 0.0000144 .9124630 .731257

C $0.0000164 .916342-0.729560$

C $0.0001697 .381931-0.717882$

C -0.000050 3.668924 1.426331

C -0.000041 3.677944-1.430983

C -0.000094 2.440704-0.736985

C -0.0001012 .4379390 .727527$

C -0.000143 1.191524 1.421019

H -0.000138 1.1921742 .509008

C -0.000164 -0.033069 0.722161

C -0.000149-0.033740 -0.737416

C $-0.0001211 .202453-1.435307$

H -0.000041 3.6689302 .514047

H 0.0001006 .1468252 .517673

H $0.0000986 .160947-2.509453$

$\mathrm{H}-0.0000273 .682145-2.518579$

H $-0.0001001 .203257-2.523006$

C - $0.000142-1.276866-1.434798$

C $-0.000182-1.2934101 .418735$

C $-0.000169-2.4956160 .726765$

C $-0.000142-2.504814-0.736106$

$\mathrm{H}-0.000184-1.2814582 .507385$

H $-0.000113-1.274565-2.522793$

C $-0.000095-3.752929-1.431524$

C $-0.000161-3.7822231 .431978$

C -0.000109-4.9705130.753182

C $-0.000066-4.989100-0.736026$

H $-0.000059-3.750514-2.519415$

H -0.000177 -3.752163 2.520337

C $0.000020-6.210588-1.420597$

C $-0.000077-6.2448271 .457141$

C $0.000077-7.488305-0.720228$ 
C $0.000018-7.4784610 .766531$

H $0.000064-6.228050-2.508064$

H -0.000111 -6.232040 2.543491

C $0.0000896 .157442-1.421524$

C $0.000268-9.959232-0.702519$

C $0.000201-9.9612230 .751735$

C $0.000195-8.677224-1.403558$

H $0.000251-8.701761-2.492156$

C $0.000073-8.6998561 .456746$

H $0.000034-8.7148922 .544302$

C $0.000272-11.1834731 .443061$

C $0.000416-12.4167780 .737200$

C $0.000487-12.410848-0.702827$

C $0.000407-11.168506-1.399648$

H $0.000466-11.173379-2.488249$

H $0.000225-11.1930182 .531396$

C $0.000721-14.857474-0.711889$

H $0.000842-15.811904-1.235474$

C $0.000649-14.8641310 .725554$

H $0.000716-15.8233371 .240258$

C $0.000642-13.673245-1.406537$

C $0.000500-13.6865621 .430370$

H $0.000698-13.657120-2.494825$

H $0.000447-13.6793242 .518737$

C $0.0002608 .654104-1.409496$

C $0.0003519 .832751-0.704939$

H $0.00042410 .791413-1.220548$

C 0.0002678 .6458561 .431720

H 0.0002738 .6329492 .520068

C 0.0003559 .8285800 .733917

H 0.00043110 .7842421 .255078

H $0.0002618 .647375-2.497892$

8 ( $n=12$, singlet $)$

C -0.002203 7.4621340 .757338

C - 0.0038506 .2180401 .452299

C - 0.0048684 .9839620 .754201

C -0.004551 4.985873 -0.712631

C -0.001756 7.464047 -0.709306

C -0.0059883 .7434051 .448840$

C -0.005511 3.747129-1.410501

C $-0.0064072 .508697-0.715383$

C - 0.0065752 .5067880 .750499

C -0.0070871 .2676861 .445380$

H - 0.0072511 .2661862 .533129

C -0.0071860 .0305220 .747079$

C -0.007176 $0.032430-0.718411$
C -0.006912 $1.271407-1.413489$

$\mathrm{H}-0.0062583 .7420842 .536557$

H -0.004209 6.218037 2.539932

H - $0.0028846 .224602-2.495141$

H -0.005416 3.748641 -2.498219

H -0.006942 $1.272739-2.501238$

C -0.007179 -1.204734-1.416712

C - $0.007043-1.2084551 .442157$

C -0.006624 -2.445745 0.744051

C - $0.006773-2.443836-0.721830$

H -0.007092 -1.209787 2.529906

H $-0.007332-1.203233-2.504461$

C -0.006253 -3.680453 - 1.420172

C $-0.005813-3.6841781 .439169$

C - $0.004876-4.9229210 .741299$

C $-0.005175-4.921010-0.725533$

H - $0.006523-3.679131-2.507889$

H $-0.005746-3.6856902 .526887$

C $-0.004145-6.155087-1.423632$

C -0.003427 -6.158817 1.436178

C $-0.002438-7.399181-0.728672$

C $-0.002009-7.4010950 .737973$

H $-0.004511-6.155084-2.511265$

H -0.003246 -6.161651 2.523808

C - $0.0030986 .221768-1.407511$

C $0.001291-9.874855-0.728752$

C $0.001817-9.8767630 .731586$

C $-0.000940-8.629710-1.425843$

H $-0.001365-8.632120-2.513407$

C $0.000003-8.6334431 .431929$

H $0.000297-8.6386932 .519484$

C $0.004282-11.1138361 .425132$

C $0.006345-12.3414810 .722945$

C $0.005761-12.339584-0.726560$

C $0.003183-11.110108-1.425530$

H $0.002726-11.110417-2.513356$

H $0.004670-11.1169902 .512953$

C $0.010673-14.789779-0.724017$

H $0.012405-15.745728-1.244222$

C $0.011280-14.7916610 .713986$

H $0.013463-15.7489691 .231685$

C $0.007968-13.607545-1.423731$

C $0.009152-13.6112631 .416794$

H $0.007475-13.596610-2.511893$

H $0.009574-13.6031762 .504982$

C $0.0001518 .696394-1.403263$

C $0.0017789 .939715-0.702921$ 
C -0.0008258 .6926631 .454509$

H - 0.0012458 .6950742 .542073

C 0.0012359 .9378080 .757417

H $0.0004768 .701643-2.490818$

C $0.00402811 .176788-1.396468$

C 0.00289711 .1730621 .454194

C 0.00521412 .4025380 .755222

C $0.00581412 .404433-0.694283$

C $0.00831913 .674215-1.388133$

C $0.01012314 .854615-0.685327$

C 0.00710413 .6705001 .452392

C 0.00950014 .8527340 .752677

H $0.00876413 .666128-2.476321$

H $0.00444111 .179941-2.484289$

H 0.00244111 .1733722 .542019

H 0.00661113 .6595672 .540554

H $0.01205915 .811923-1.203026$

H 0.01097515 .8086841 .272881

$8(n=12$, triplet $)$

C 0.0000767 .4638230 .761673

C - 0.0002756 .2228161 .459623

C 0.0001504 .9845320 .764394

C $0.0009814 .983145-0.703086$

C $0.0009117 .462140-0.705496$

C -0.000222 3.749290 1.461432

C $0.0013923 .745025-1.397907$

C $0.0009982 .506075-0.699976$

C 0.0001752 .5065940 .764510

C -0.0002241 .2732891 .460655$

H -0.0008281 .2702452 .548288$

C 0.0001390 .0332070 .760703

C $0.0009530 .035399-0.697469$

C $0.0013751 .267578-1.395213$

H -0.000831 3.748154 2.549022

H - 0.0008886 .2247482 .547193

H $0.0019706 .218935-2.488272$

H $0.0020113 .743138-2.485590$

H $0.0019891 .267070-2.483079$

C $0.001291-1.217702-1.395759$

C -0.000291 -1.207444 1.456575

C $0.000035-2.4360310 .755622$

C $0.000842-2.426356-0.703847$

H -0.000889 - 1.2079262 .544419

H $0.001900-1.207362-2.484162$

C $0.001144-3.708251-1.410387$

C -0.000429 -3.686271 1.450432
C -0.000151 -4.9167630.753013

C $0.000667-4.896893-0.732094$

H $0.001753-3.678137-2.498714$

H $-0.001020-3.6840072 .538222$

C $0.000932-6.175113-1.439716$

C $-0.000645-6.1457951 .438023$

C $0.000400-7.403767-0.753126$

C - $0.000417-7.4172740 .736792$

H $0.001547-6.156108-2.525911$

H -0.001229 -6.161018 2.525212

C $0.0013486 .219033-1.400658$

C $0.000039-9.890698-0.742601$

C -0.000754 -9.891709 0.713546

C $0.000614-8.628025-1.445429$

H $0.001216-8.640168-2.532665$

C $-0.000947-8.6091021 .417178$

H $-0.001539-8.6362682 .505490$

C -0.001325 -11.099230 1.407697

C $-0.001154-12.3435820 .707906$

C $-0.000375-12.347030-0.730658$

C $0.000218-11.109817-1.435288$

H $0.000809-11.119292-2.523465$

H -0.001915 -11.108199 2.496152

C $-0.000817-14.793616-0.723003$

H - $0.000711-15.751160-1.240373$

C -0.001592-14.7893190.712854

H -0.002047 -15.743763 1.235989

C $-0.000223-13.613662-1.426179$

C -0.001755 -13.604942 1.409758

H $0.000366-13.605351-2.514338$

H -0.002341 -13.591416 2.497881

C $0.0012578 .693130-1.402349$

C $0.0007989 .938254-0.705096$

C -0.0003738 .6967481 .455687$

H -0.000990 8.7018452 .543223

C -0.0000369 .9400350 .755428$

H $0.0018808 .695725-2.489910$

C $0.00113011 .173378-1.401708$

C -0.000501 11.176938 1.449019

C -0.000172 12.4048870.746836

C $0.00065812 .403090-0.702560$

C $0.00097913 .670876-1.399651$

C $0.00050214 .853229-0.699815$

C -0.000649 13.674414 1.440778

C - 0.00032214 .8550180 .738006

H $0.00160313 .660107-2.487818$

H $0.00175411 .173944-2.489533$ 


\section{Section3: Table S3}

Table S3: Total energies (in a.u.) of singlet $\left(E_{\mathrm{S}}\right)$, triplet $\left(E_{\mathrm{T}}\right)$, triplet state at the singlet geometry $\left(E_{\mathrm{T}^{\prime}}\right)$, the total energy after the $\mathrm{AP}$ correction $\left(E_{\mathrm{AP}}\right)$ and the corresponding expectation values of total spin angular momentum $\left(\left\langle\mathbf{S}^{2}\right\rangle_{\mathrm{S}}\right.$ and $\left.\left\langle\mathbf{S}^{2}\right\rangle_{\mathrm{T}^{\prime}}\right)$ (in a.u.) for the three isomers of benzyne calculated by UAM1, UPM3, UPM6, UrPM6, and UB3LYP.

\begin{tabular}{|c|c|c|c|c|c|c|}
\hline & $E_{\mathrm{S}}$ & $E_{\mathrm{T}^{\prime}}$ & $\left\langle\mathbf{S}^{2}\right\rangle_{\mathrm{S}}$ & $\left\langle\mathbf{S}^{2}\right\rangle_{\mathrm{T}^{\prime}}$ & $E_{\mathrm{AP}}$ & $E_{\mathrm{T}}$ \\
\hline \multicolumn{7}{|c|}{ ortho-benzyne } \\
\hline UAM1 & 0.199098 & 0.219805 & 1.4050 & 2.0163 & 0.151504 & 0.217580 \\
\hline UPM3 & 0.188088 & 0.216734 & 1.3251 & 2.0249 & 0.133844 & 0.212546 \\
\hline UPM6 & 0.190235 & 0.210930 & 1.0025 & 2.0086 & 0.169615 & 0.206899 \\
\hline UrPM6 & -0.009331 & 0.012920 & 0.6042 & 2.0049 & -0.018929 & 0.004534 \\
\hline $\mathrm{UB} \mathrm{LYP}^{a}$ & -230.909939 & & 0.0000 & & & -230.862994 \\
\hline \multicolumn{7}{|c|}{ meta-benzyne } \\
\hline UAM1 & 0.203993 & 0.201170 & 0.9487 & 2.5593 & 0.205656 & 0.200773 \\
\hline UPM3 & 0.196890 & 0.190618 & 0.9865 & 2.5860 & 0.200759 & 0.190054 \\
\hline UPM6 & 0.189497 & 0.192709 & 0.9201 & 2.2855 & 0.187333 & 0.192195 \\
\hline UrPM6 & -0.018900 & -0.002268 & 0.7763 & 2.0505 & -0.029033 & -0.004277 \\
\hline UB3LYP & -230.888143 & -230.856039 & 0.4818 & 2.0195 & -230.898202 & -230.869519 \\
\hline \multicolumn{7}{|c|}{ para-benzyne } \\
\hline UAM1 & 0.195262 & 0.207618 & 1.5749 & 2.0214 & 0.151678 & 0.207254 \\
\hline UPM3 & 0.186323 & 0.198798 & 1.6188 & 2.0344 & 0.137730 & 0.198444 \\
\hline UPM6 & 0.188843 & 0.195475 & 1.3262 & 2.0116 & 0.176009 & 0.195244 \\
\hline UrPM6 & -0.009624 & -0.003505 & 1.0025 & 2.0056 & -0.015739 & -0.004051 \\
\hline UB3LYP & -230.875598 & -230.870946 & 0.9533 & 2.0062 & -230.879811 & -230.871699 \\
\hline
\end{tabular}

${ }^{a}$ The AP method was not used because the closed-shell singlet is obtained. 


\section{Section4: Table S4}

Table S4: Total energies (in a.u.) of singlet $\left(E_{\mathrm{S}}\right)$, triplet $\left(E_{\mathrm{T}}\right)$, triplet state at the singlet geometry $\left(E_{\mathrm{T}^{\prime}}\right)$, the total energy after the AP correction $\left(E_{\mathrm{AP}}\right)$ and the corresponding expectation values of total spin angular momentum $\left(\left\langle\mathbf{S}^{2}\right\rangle_{\mathrm{S}}\right.$ and $\left.\left\langle\mathbf{S}^{2}\right\rangle_{\mathrm{T}^{\prime}}\right)$ (in a.u.) for meta-xylylene calculated by UAM1, UPM3, UPM6, UrPM6, and UB3LYP.

\begin{tabular}{lcccccc}
\hline & $E_{\mathrm{S}}$ & $E_{\mathrm{T}^{\prime}}$ & $\left\langle\mathbf{S}^{2}\right\rangle_{\mathrm{S}}$ & $\left\langle\mathbf{S}^{2}\right\rangle_{\mathrm{T}^{\prime}}$ & $E_{\mathrm{AP}}$ & $E_{\mathrm{T}}$ \\
\hline meta-xylylene & & & & & & \\
\hline UAM1 & 0.107513 & 0.087951 & 1.1914 & 2.5319 & 0.124900 & 0.085249 \\
UPM3 & 0.106968 & 0.088855 & 1.1580 & 2.5256 & 0.122304 & 0.085904 \\
UPM6 & 0.106655 & 0.090332 & 1.1123 & 2.4406 & 0.120324 & 0.087518 \\
UrPM6 & -0.110307 & -0.124623 & 1.0204 & 2.2288 & -0.098217 & -0.127037 \\
UBHandHLYP & -309.377813 & -309.393346 & 1.0209 & 2.1677 & -309.363986 & -309.395157 \\
UB3LYP & -309.574418 & -309.584411 & 1.0124 & 2.0682 & -309.564836 & -309.585124 \\
\hline
\end{tabular}

\section{Section5: Full citation for Ref. 65}

(65) Frisch, M. J.; Trucks, G. W.; Schlegel, H. B.; Scuseria, G. E.; Robb, M. A.; Cheeseman, J. R.; Scalmani, G.; Barone, V.; Mennucci, B.; Petersson, G. A.; Nakatsuji, H.; Caricato, M.; Li, X.; Hratchian, H. P.; Izmaylov, A. F.; Bloino, J.; Zheng, G.; Sonnenberg, J. L.; Hada, M.; Ehara, M.; Toyota, K.; Fukuda, R.; Hasegawa, J.; Ishida, M.; Nakajima, T.; Honda, Y.; Kitao, O.; Nakai, H.; Vreven, T.; Montgomery, J. A., Jr.; Peralta, J. E.; Ogliaro, F.; Bearpark, M.; Heyd, J. J.; Brothers, E.; Kudin, K. N.; Staroverov, V. N.; Kobayashi, R.; Normand, J.; Raghavachari, K.; Rendell, A.; Burant, J. C.; Iyengar, S. S.; Tomasi, J.; Cossi, M.; Rega, N.; Millam, J. M.; Klene, M.; Knox, J. E.; Cross, J. B.; Bakken, V.; Adamo, C.; Jaramillo, J.; Gomperts, R.; Stratmann, R. E.; Yazyev, O.; Austin, A. J.; Cammi, R.; Pomelli, C.; Ochterski, J. W.; Martin, R. L.; Morokuma, K.; Zakrzewski, V. G.; Voth, G. A.; Salvador, P.; Dannenberg, J. J.; Dapprich, S.; Daniels, A. D.; Farkas, Ö.; Foresman, J. B.; Ortiz, J. V.; Cioslowski, J.; Fox, D. J. Gaussian 09, Revision D.01; Gaussian, Inc.; Wallingford CT, 2009. 